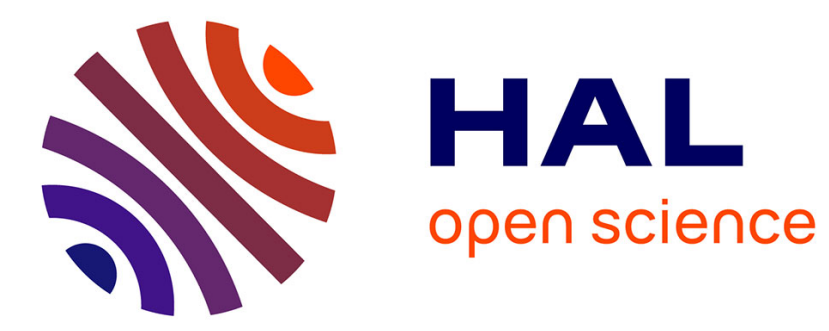

\title{
Photometric reconstruction of a dynamic textured surface from just one color image acquisition
}

Benjamin Bringier, David Helbert, Majdi Khoudeir

\section{To cite this version:}

Benjamin Bringier, David Helbert, Majdi Khoudeir. Photometric reconstruction of a dynamic textured surface from just one color image acquisition. Journal of the Optical Society of America. A Optics, Image Science, and Vision, 2008, 25 (3), pp.566-574. 10.1364/JOSAA.25.000566 . hal-00331323

\section{HAL Id: hal-00331323 \\ https://hal.science/hal-00331323}

Submitted on 17 Oct 2008

HAL is a multi-disciplinary open access archive for the deposit and dissemination of scientific research documents, whether they are published or not. The documents may come from teaching and research institutions in France or abroad, or from public or private research centers.
L'archive ouverte pluridisciplinaire HAL, est destinée au dépôt et à la diffusion de documents scientifiques de niveau recherche, publiés ou non, émanant des établissements d'enseignement et de recherche français ou étrangers, des laboratoires publics ou privés. 


\title{
The photometric Reconstruction of a Dynamic Textured Surface from just one Colour Image Acquisition 1
}

\author{
Benjamin Bringier, David Helbert* and Majdi Khoudeir \\ SIC Laboratory, University of Poitiers, BP 30179 \\ 86962 Futuroscope-Chasseneuil Cedex, France \\ *Corresponding author: helbert@sic.sp2mi.univ-poitiers.fr
}

\begin{abstract}
Textured surface analysis is essential for many applications. In this paper, we present a three-dimensional recovery approach for real textured surfaces based on photometric stereo. The aim is to be able to measure the textured surfaces with a high degree of accuracy. For this, we use a color digital sensor and principles of color photometric stereo. This method uses a single color image, instead of a sequence of gray-scale images, to recover the surface of the three dimensions. It can thus be integrated into dynamic systems where there is significant relative motion between the object and camera. To evaluate the performances of our method, we compare it, on real textured surfaces to traditional photometric stereo using three images. We show thus that it is possible to have similar results with just one color image. (C) 2008 Optical Society of America
\end{abstract}

\section{Introduction}

The three-dimensional analysis of textured surfaces by non-contact recovery methods is not commonplace. Nevertheless, there are many surface recovery applications: fault detection, surface inspection, face recognition... Nowadays, the most powerful method is to acquire the surface using a laser system [1]. Indeed, this method, known as the direct method, measures range by estimating how long it takes light to reach the target and return to the sensor. It allows an analysis with a high degree of accuracy. However, this method has many disadvantages. For relatively small surfaces (a few square centimeters), acquisition times are

${ }^{1}$ The electronic version of this article can be found online at
http://josaa.osa.org/abstract.cfm?msid=85528


very long and the system cost is high. It is thus not possible to integrate this method into dynamic systems or for relatively high acquisition rates.

Image processing makes it possible to develop other methods known as indirect methods. They estimate distance by measuring parameters calculated from the image intensity of the illuminated object. Several categories of methods are employed. The first, stereovision [2] uses triangulation from several acquisitions of the same scene made by several cameras to compute depth. The problem with this method is that it requires a relationship between points in one image and the same points in another image, especially for textured surfaces. The second, optical flow [3] calculates the relative distance to points on the surface of an object by analysing how image points flow from one frame to the next. The problem here is to make two images correspond in order to obtain an accurate analysis. The last method, shape from shading measures the surface shape by analysing the radiometry of image formation [4]. As shown in various studies [5], this method is not precise but has the advantage of using only one image. To improve the results, several acquisitions of the same differently illuminated scene are used [6]. This method is called photometric stereo and was largely studied these last decades [7-9]. For very short acquisition times and large surfaces, the results of three-dimensional surface analysis then approach the accuracy of the laser methods $[10,11]$. However, relative motion between the camera and the object poses a problem for photometric stereo derived from a sequence of images in a dynamic environment. Indeed, the camera has to remain static for the various acquisitions. Several solutions are suggested in the literature to solve this problem. Pentland [12] employs both reflectance maps and optical flow information for the estimation of shape from image sequences of dynamic scenes. Malsbender in [13] uses a camera and several LEDs. The alternative illumination of the LED is synchronized with the camera frequency. These must then be much faster than the displacement frequency of the scene studied. Lastly, multispectral approaches are proposed by Drew [14] or by Smith [15]. The first presents a method for recovering the shape of a Lambertian surface with unknown but uniform reflectance from a single composite RGB image. In [16], Drew proposes a calibration method using a lookup table based on a sphere. Smith et al. present a technique termed narrow infrared photometric stereo. For these two approaches, a camera with good spectral channel isolation is necessary.

We propose in this article to use the color approach within the meaning of [14] for threedimensional textured surface data capture. The theory of this system is simple, the gray images in case of photometric stereo are replaced by the three sensor channels (red, green and blue). To each of the sensor's colors or spectral channels, must correspond an illumination of the same color or spectral band. The principles of photometric stereo are then applied to extract three-dimensional information. Unfortunately, in practice it is difficult to obtain perfect spectral channel isolation with a simple color camera. Indeed, the three channels use 
a common part of the spectrum [17]. Moreover, the surfaces studied must have a known uniform colour. This assumption will be checked in the remainder of this article. For this approach, a precise spectral characterization is thus necessary to obtain similar performances to the photometric stereo with three images. This spectral characterization of each part of the acquisition system is proposed in this article. And, we can then compensate for effects

such as spectral correlation. The system obtained is inexpensive and can be used to analyse dynamic textured surfaces.

This article is broken down into three distinct parts. The first one introduces the theoretical approach of photometric stereo and formulates the color photometric stereo using just one image. The second one shows that in theory the two methods give the same results if the acquisition system is perfect. For that, a textured surface base acquired by a laser system is used. The last part proposes the real system. In this part, we carry out the spectral characterization of the system and propose a correction method for spectral correlation. We evaluate the performances of our method on real textured surfaces by comparing it with three image photometric stereo.

\section{The theoretical approach of photometric stereo}

\section{A. The Lambertian model}

In this study, surfaces to be reconstructed are considered as purely diffuse. The simplest model, formulated by Lambert in 1760 [18], is the quantitative law for perfectly diffuse surfaces. Lambert stated that a perfectly diffuse surface appears equally bright from all viewing directions. He assumed that the diffuse surface has a homogeneous reflectance function. He ignored self or cast shadows, as well as any inter-reflection. Under these assumptions and for a point light source at infinity, the reflected intensity from one point on the surface is proportional to the angle between the surface normal at that point and the illuminant direction:

$$
I=I_{0} \rho \cos (\theta)=I_{0} \rho(\mathbf{N} \cdot \mathbf{L})
$$

or in scalar form:

$$
i(x, y)=i_{0} \rho(x, y) \frac{-p \cos \tau \sin \sigma-q \sin \tau \sin \sigma+\cos \sigma}{\sqrt{p^{2}+q^{2}+1}}
$$

where:

- $I$ or $i(x, y)$ is the intensity reflected at the point $(x, y)$,

- $I_{0}$ or $i_{0}$ is the incident intensity,

- $\rho(x, y)$ is the albedo; a coefficient that represents the proportion of light reflected from the point $(x, y)$ with respect to the incident light, 
- $\mathbf{N}=\left(\frac{-p}{\sqrt{p^{2}+q^{2}+1}}, \frac{-q}{\sqrt{p^{2}+q^{2}+1}}, \frac{1}{\sqrt{p^{2}+q^{2}+1}}\right)$ is the unit vector normal to the surface at $(x, y)$,

- $\mathbf{L}=(\cos \tau \sin \sigma, \sin \tau \sin \sigma, \cos \sigma)$ is the unit vector pointing at the light source,

- $\tau$ and $\sigma$ are the light azimuth and zenith defined as in figure 1

- $\theta$ is the angle between the normal to the surface at $(x, y)$ and the illuminant direction,

- $p=\frac{\partial S(x, y)}{\partial x}$ and $q=\frac{\partial S(x, y)}{\partial y}$ represent the partial derivatives with respect to $x$ and $y$ of the surface $S(x, y)$.

\section{B. Determination of a gradient field}

If, in equation 2, $i_{0}$ is considered as constant on all the surface analyzed. As Woodham has shown in [6], parameters $\rho, p$ and $q$ can be determined from three acquisitions of the same scene under three angles of different illumination incidence. In fact, a system of three equations with three unknowns can be defined:

$$
i_{k}(x, y)=\rho(x, y) i_{0} \frac{p S_{k x}+q S_{k y}-S_{k z}}{\sqrt{p^{2}+q^{2}+1}} \text { with } k=1,2,3
$$

with $S_{k x}=-\cos \tau_{k} \sin \sigma, S_{k y}=-\sin \tau_{k} \sin \sigma$ and $S_{k z}=\cos \sigma$.

\section{C. Reconstruction from a gradient field}

Various algorithms exist to obtain a tridimensional reconstruction from a gradient field [5, 19-24]. For our application, we use the Frankot-Chellappa algorithm to integrate the partial derivatives [25].

Frankot and Chellappa introduced in [25] an algorithm to recover the surface, in using the Fourier transform applied to the gradient field. This method is currently used in various published works and it has the advantage of being robust to noise [26]. Moreover, fast Fourier transform allows very fast computation times for large size images (less than one second on a current computer for a surface of 512 by 512 pixels). The algorithm minimizes a least square error function defined by the following equation:

$$
W=\iint\left(\frac{-\partial s(x, y)}{\partial x}-p\right)^{2}+\left(\frac{-\partial s(x, y)}{\partial y}-q\right)^{2} d x d y
$$

with $s(x, y)$ the original surface. In applying Perseval's theorem, the expression which relates the Fourier transform of the surface $Z(u, v)$ and those of these Fourier tranform of the gradients $P(u, v)$ and $Q(u, v)$ is given by:

$$
S(u, v)=\frac{-j u P(u, v)-j v Q(u, v)}{u^{2}+v^{2}}
$$


with $(u, v) \neq(0,0)$.

From this very simple relation, the reconstructed surface is directly obtained by inverse Fourier transform of $S$. However, this technique is limited to the reconstruction of surfaces whose gradient average value is null $(\hat{p}(0,0)=0$ and $\hat{q}(0,0)=0)$ since we cannot process the cases where $(u, v)=(0,0)$. In other words, the mean plane of the surface must be null.

\section{The theoretical approach for color photometric stereo}

One of the principal defects of photometric stereo is the obligation to acquire the same scene several times. The latter cannot thus be mobile, which poses many problems for industrial applications. Knowing that the use of digital color sensors is very widespread today, the most obvious solution a priori is to process the colour information as the generation of three spectral images of the same scene. The solution is to illuminate the surface with three isolated spectral lights. However, this solution is not so commonplace and certain hypotheses must be respected to obtain satisfactory results. Firstly, except for Foveon X3 sensors (The Foveon $\mathrm{X} 3$ sensor is an image sensor for digital cameras, designed by Foveon inc. It uses an array of photosites, each of which consists of three vertically stacked photodiodes, that are organized in a horizontal and vertical grid) or the tri-CCD (Charged Coupled Device) cameras, a Bayer filter (figure 2) is used in front of the photosensitive sensor to separate information spectrally. The result is that a point of the image is represented by just one red, green or blue spectral band. For a facet of surface, a red, green and blue triplet must correspond.

In the second place and this is surely the most important hypothesis, the information between the three spectral channels must be completely isolated in order to be able to find the normal of each facet. The choice of light sources is thus of primary importance. Lastly, the surfaces analyzed must reflect the light over all the visible spectrum, otherwise, only one or two images can be used for the reconstruction. However, it is rather rare in nature to find

surfaces only emit light in one part of the visible spectrum. Nevertheless, a small surface reflection on one of the spectral bands of the sensor can introduce a loss of information.

\section{A. The principle of Color photometric stereo}

The principle of color photometric stereo is very simple. A surface is illuminated by three isolated spectral sources of light : a red one or long spectral band, a green one or mean spectral band and a blue one or short spectral band. The spectral radiance distribution reflected by each facet of the surface changes according to normal facet direction. Figure 3 presents the example of a computer-created half-sphere lighted by three isolated colored sources of light. Now, the RGB sensor creates three spectral images which correspond to the three different illuminations. The photometric stereo theory can be applied on these images to recover the $3-\mathrm{D}$ surface. 
A schematic configuration of the physical set-up is shown in figure 4

\section{B. The mathematical approach}

The color of an object is strongly dependent on its spectral reflectance, that is, the amount of incident light that is reflected from the surface for different wavelengths. If we represent the spectral radiance of the illuminant by the function $I_{0}(\lambda), \lambda$ being the wavelength, and the spectral reflectance in a given surface point of the object by $\rho(\lambda)$, the radiance of light reflected from this surface point $I(\lambda)$ is, by definition of spectral reflectance :

$$
I(\lambda)=I_{0}(\lambda) \rho(\lambda)
$$

Now, we can rewrite Lambert's law by integrating spectral information:

$$
I(\lambda)=I_{0}(\lambda) \rho(\lambda)(\mathbf{N} \cdot \mathbf{L})
$$

And, if we place a color filter in front of the light source to conserve a part of the spectral radiance of the light, $I(\lambda)$ becomes $I_{c}(\lambda)$ :

$$
I_{c}(\lambda)=I_{0}(\lambda) \rho(\lambda) F_{c}(\lambda)\left(\mathbf{N} \cdot \mathbf{L}_{\mathbf{c}}\right)
$$

where $F_{c}(\lambda)$ is the color filter placed in front of the light source and $c$ represents the color of the filter. In this case, $c$ are for the red $r$, green $g$ and blue $b$ color filters.

In the three equation system, to determine the gradient's surface $p$ and $q$, we have to integrate the spectral sensitivity of the three acquisition sensor channels. We define $C_{r}(\lambda), C_{g}(\lambda)$ and $C_{b}(\lambda)$ that represent the red, green and blue sensitivity of the sensor. The equation system is given by:

$$
\left\{\begin{array}{l}
I_{1}(\lambda)=I_{r, g, b} C_{r}(\lambda) \\
I_{2}(\lambda)=I_{r, g, b} C_{g}(\lambda) \\
I_{3}(\lambda)=I_{r, g, b} C_{b}(\lambda)
\end{array}\right.
$$

where $I_{r, g, b}$ defines the light reflected from the surface, illuminated by three illuminants:

$$
\begin{aligned}
I_{r, g, b} & =I_{0}(\lambda) \rho(\lambda) \\
& \times\left(F_{r}(\lambda)\left(\mathbf{N} \cdot \mathbf{L}_{\mathbf{r}}\right)+F_{g}(\lambda)\left(\mathbf{N} \cdot \mathbf{L}_{\mathbf{g}}\right)+F_{b}(\lambda)\left(\mathbf{N} \cdot \mathbf{L}_{\mathbf{b}}\right)\right)
\end{aligned}
$$

where $\mathbf{L}_{\mathbf{r}}, \mathbf{L}_{\mathbf{g}}$ and $\mathbf{L}_{\mathbf{b}}$ are the unit vectors pointing respectively at the red, green and blue light

source. If, $\left(C_{r}, F_{r}\right),\left(C_{g}, F_{g}\right)$ and $\left(C_{b}, F_{b}\right)$ are decorrelated and $\rho(\lambda)$ does not vary according to $\lambda, I_{1}, I_{2}$ and $I_{3}$ are same equations as in the standard photometric stereo with three images, introduced in section 2.C. 


\section{The simulation of $3 \mathrm{D}$ reconstruction of textured surfaces}

The method of 3D reconstruction is now applied to surfaces known as textured (road coating). Initially, surfaces acquired by the laser system are used to simulate images [11]. Surface size is $40.96 \mathrm{~mm} \times 40.96 \mathrm{~mm}$ with a resolution of $80 \mu \mathrm{m}$ in $\mathrm{X}$ and $\mathrm{Y}$. The resolution in $\mathrm{Z}$ is $1 \mu \mathrm{m}$ and the high variation in $\mathrm{Z}$ is $1 \mathrm{~cm}$. This base proposes very different examples which are based on real textures. The geometric elements of which they are made up have very varied structures and sizes. The basic surfaces used for simulation are presented in figure 5 . The objective is to measure distortion introduced by the reconstruction method. Then, always by simulation, this algorithms robustness with noise is evaluated.

\section{A. Method}

In this paper, all the simulations use the same framework. First, gradient fields are calculated from surfaces acquired by a laser system. Then, Lambert's model is used to predict the appearance of the surface. For each surface, three acquisitions of gray level images or one color acquisition is simulated with an angle $\sigma=\frac{3 \pi}{70}$ and an angle $\tau=\left[\frac{\pi}{3}, \pi, \frac{5 \pi}{3}\right]$. Finally, the algorithm to recover the 3-D surface, presented in the previous part 2] is also used from simulated acquisitions. Figure 6 gives the framework of the simulation.

For each 3-D reconstruction, the Signal to Noise Ratio (SNR) between the normalization of surfaces from the base and reconstructed surfaces is computed:

$$
S N R=10 \log \left(\frac{\frac{1}{m n} \sum_{i=0}^{m-1} \sum_{i=0}^{m-1} S(i, j)^{2}}{M S E}\right)
$$

where $M S E$ is the mean squared error which for two $m \times n$ surfaces $s$ and $\hat{s}$ where $\hat{s}$ is considered a noisy approximation of $s$ :

$$
M S E=\frac{1}{m n} \sum_{i=0}^{m-1} \sum_{i=0}^{m-1}\|S(i, j)-\hat{S}(i, j)\|^{2}
$$

\section{B. Results without noise}

Figure[ [ ]shows an example of a textured surface illuminated by three different azimuth angles (image 1: $\tau_{1}=\frac{\pi}{3}$, image 2: $\tau_{2}=\pi$ and image 3: $\tau_{3}=\frac{5 \pi}{3}$ ) with Lambert's model. Figure 8 shows one example of a textured surface illuminated by three colored lights with different azimuth angles (the blue light: $\tau_{b}=\frac{\pi}{3}$, the green light: $\tau_{g}=\pi$ and the red light: $\tau_{r}=\frac{5 \pi}{3}$ ) by lambert's model. All cases use the same zenith $\sigma=\frac{3 \pi}{70}$.

Now, the SNR between the normalization of surfaces from the laser acquisition and reconstructed surfaces, from three images or one color image is computed (cf. table प). In all cases, reconstruction errors are small and they are exclusively at the surface edges (figure 9) or on the border of the images. These border errors are well known in image processing. 
Results for three gray level images or one color image are the same because the three color channels are considered to be decorrelated. One last remark, the images in this simulation are considered to be acquired by an eight bit sensor, in practice, professional cameras use twelve bit sensors. For the same exposure time, a twelve bit sensor will have a better definition (smaller quantum) than a eight bit sensor. Moreover, that makes it possible to recover a part of surface more effectively if it is over or under-exposed.

Figure 9] shows a part of the original textured surface (figure 5(a)) and the same part of the reconstructed surface. The reconstructed surface is the same for three gray level acquisitions or one color acquisition (figure 9). An error map is computed between these two surfaces and the SNR is $36.201 \mathrm{~dB}$.

\section{C. Results with noise}

To verify the robustness of the reconstruction algorithm, a white Gaussian noise of variance

$\sigma^{2}$ which is equal to $0.05,0.15$ and 0.3 is added to each channel of the image before reconstruction. Respectively, the SNRs between the original images and the noise version are $15.513 \mathrm{~dB}, 9.375 \mathrm{~dB}$ and $8.105 \mathrm{~dB}$. The two last values are very high and not met in real acquisitions. So these values make it possible to check the 3-D recover algorithm robustness in extreme conditions. Figure 10 illustrates the addition of this noise for three acquisitions of gray level images or one color image acquisition.

As for the 3-D reconstruction without noise, the SNR is calculated between the recovered surface with and without noise, for three gray level images or one color image. Table 2 gives the results for figure 5. SNR variations between the reconstruction without noise and with noise are very small. But, in the case of color acquisition, the SNR decreases more quickly than in the case of the three gray level acquisitions. For gray level images, the three channels can be used to reduce noise. Indeed, in this case, luminance is the result of the red, green and blue channel combination. If the noise is decorrelated between these three channels, the luminance noise is lower. If, only one channel is used, the results are similar between three image photometric stereo and one image photometric stereo.

\section{Real system}

To carry out the method of relief extraction, we use a Nikon(C D100 single lens reflex camera and a Sigma(c) 150mm EX DG F2.8 Macro lens with three gas-discharge lamps (cf. figure 11). For color acquisition, we use three band-pass color filters in front of the light, one each for small, median and long wavelengths. During our acquisitions, the acquisition system must be isolated from any external light. The chosen size of the surface analyzed is 142.7 $\mathrm{mm} \times 115.5 \mathrm{~mm}$, which subtended the entire camera field-of-view. Camera resolution was $3037 \times 2024$ pixels, resulting in a resolution of $47 \times 57 \mu \mathrm{m}$ at the object's surface. 


\section{A. Spectral characterization}

In reality, it is difficult to obtain spectral filters which separate the visible spectrum into orthogonal components. It is not thus obvious to carry out a system of photometric stereo which is perfect at spectral level. Moreover, the red and blue are superimposed on at least one other color and the green is superimposed on both the red and blue. A precise characterization of each component constituting the chain is necessary. A spectrocolorimeter PR650 of PhotoResearch is used in this study for the spectral analysis. The method which is proposed in [27] is applied with 74 patches of a MacBeth calibration chart to characterize the spectral sensitivity of the camera.

Figure 12(a) shows the spectral radiance distribution for a gas-discharge lamp used in the system of 3-D reconstruction. This distribution is not uniform but radiance is not canceled in any point. To separate the visible spectrum into orthogonal components, spectral filters are placed in front of the lights. Figure 12(b) shows the gain of these filters, one for short wavelengths, one for medium wavelengths and one for long wavelengths. The red and blue filter are correct but the green filter has a defect in the short wavelengths. Then, figure 12(c) shows the measurement of the camera's Bayer filter. The three spectral channels are not isolated which can introduce some errors into the 3-D recovered surface. Finally, if the surface reflectance is uniform, the signal received by the camera's sensor is calculated 12(d): that is the spectral multiplication of the light's spectral radiance distribution, the gain of the spectral filters (placed in front of light) and the camera's Bayer filter.

By using equations 9 and 10, the relationship between the light reflected from the surface and the images used in photometric stereo can be solved by computing:

$$
\left[\begin{array}{l}
I_{1} \\
I_{2} \\
I_{3}
\end{array}\right]=I_{0} \rho \times \Gamma \times\left[\begin{array}{l}
\left(\mathbf{N} . \mathbf{L}_{\mathbf{r}}\right) \\
\left(\mathbf{N} . \mathbf{L}_{\mathbf{g}}\right) \\
\left(\mathbf{N} . \mathbf{L}_{\mathbf{b}}\right)
\end{array}\right]
$$

where $\Gamma$ is the correlation between spectral filters in front of the lights and the camera's Bayer filter and is defined by:

$$
\Gamma=\left[\begin{array}{ccc}
C_{r} \times F_{r} & C_{r} \times F_{g} & C_{r} \times F_{b} \\
C_{g} \times F_{r} & C_{g} \times F_{g} & C_{g} \times F_{b} \\
C_{b} \times F_{r} & C_{b} \times F_{g} & C_{b} \times F_{b}
\end{array}\right]
$$

If the spectral channels are perfectly isolated, the $\Gamma$ is 3 -by-3 unit matrix. The results for our system are:

$$
\Gamma=\left[\begin{array}{lll}
1.000 & 0.236 & 0.042 \\
0.073 & 1.000 & 0.139 \\
0.058 & 0.033 & 1.000
\end{array}\right]
$$


The maximum level of inter-channel correlation is 0.236 . This matrix is used in 3-D recovered surface computation to correct the spectral defects of the physical system. The use of this matrix makes it possible to increase approximately $10 \mathrm{~dB}$ for the SNR between photometric stereo with three images and color photometric stereo.

\section{B. Results}

The images in figure 13 show two examples of the real textured surfaces used. The first is a part of a road and the second a part of stucco. The camera shutter speed is $1 / 30$ second and the aperture is focal/9. The photometric stereo with three images and the color photometric stereo is applied to these surfaces to recover the 3-D surface.

Figure 14 shows the results for the road surface. The SNR between the three image recovered surface and one image recovered surface is $28.052 \mathrm{~dB}$. We can see that the hollows are not so well reconstructed in the case of the color photometric stereo. This is due to the fact that the light intensity is divided by three for each azimuth angle. A zoom on part of

the surface shows this phenomenon well. However, the results of 3-D reconstruction are very close in both cases as the SNR shows.

Figure [15] shows the results for stucco. The SNR between three image recovered surfaces and one image recover surface is $47.168 \mathrm{~dB}$. As the surface is lighter, we do not find the defects of the first surface.

The 3D reconstruction results between a standard photometric stereo and our color photometric stereo are thus similar. Indeed, the SNR between the two systems is very good but can still be improved. The use of more precise spectral filters in front of the lights or a camera with better isolated spectral channels, would give even more accurate results.

\section{Conclusion and perspectives}

We have introduced an approach to obtain a surface height map from an image lighted by three colored lamps. The extraction of the height map is made with photometric stereo. The aim of our work is to be able to reconstruct the 3-D surface for the dynamic analysis of textures. The accuracy evaluation of our method is made with simulations and real acquisitions. We prove that it is possible to obtain similar results with our method to those of the classical photometric stereo. This method is not expensive and makes it possible to quickly analyze large dimension surfaces with good precision.

From this photometric stereo technique, we are currently developing approaches which take into account the specular micro-facets [28] and which explore the generalization of the Lambertian model [29] allowing to consider these various phenomena and to lead to a relief extraction by photometric stereo [30]. Our method enables efficient 3-D surface reconstruction from images acquired in a dynamic environment, i.e. with significant relative 
motion between the object and camera.

\section{Acknowledgements}

The authors are indebted to the ANR's DIVAS project for funding.

\section{References}

1. H. Zahouani, R. Vargiolu, and M.-T. Do, "Characterization of micro texture related to wet road/tire friction," in "AIPCR/PIARC," (2000), pp. 195-205.

2. O. Faugeras, Three-dimensional computer vision: a geometric view-point (Cambridge M A., 1995).

3. B. Shahraray and M. Brown, "Robust depth estimation from optical flow," IEEE Comput. Soc. Press: Second International Conference on Computer Vision pp. 641-650 (1988).

4. B. Horn, "Shape from shading : A method for obtaining the shape of a smooth opaque object from one view," Ph.D. thesis, Massachusetts Institute of Technology, Cambridge (1970).

5. R. Zhang, P. Tsai, J. Cryer, and M. Shah, "Shape from shading: a survey," IEEE Transactions on Pattern Analysis and Machine Intelligence 21, 690-706 (1999).

6. R. Woodham, "Photometric method for determining surface orientation from multiple images," Optical engineering 19, 139-144 (1980).

7. J. E. N. Coleman and R. C. Jain, "Obtaining 3-dimensional shape of textured and specular surface using four-source photometry," Computer Graphics Image Processing 18, 309-328 (1982).

8. K. Ikeuchi, "Determining surface orientations of specular surfaces by using the photometric stereo method," IEEE Transaction on Pattern Analysis and Machine Intelligence 3, 141-184 (1981).

9. G. McGunnigle and M. Chantler, "Rough surface description using photometric stereo," Measurement Science and Technology pp. 699-709 (2003).

10. A. Ben Slimane, M. Khoudeir, J. Brochard, V. Legeay, and M.-T. Do, "Relief reconstruction of rough textured surface through image analysis." in "M-T.IS\&T/SPIE 15Tth annual symposium on Electronic Imaging Science and technology," (Santa Clara, California, USA, 2003).

11. M. Khoudeir, J. Brochard, A. Benslimane, and M.-T. Do, "Estimation of the luminance map for a lambertian photometric model: application to the study of road surface roughness." Journal of Electronic Imaging 3, 512-522 (2004).

12. A. Pentland, "Photometric motion," 13(9), 879-890 (1991). 
13. T. Malzbender, B. Wilburn, D. Geld, and B. Ambrisco, "Surface enhancement using real-time photometric stereo and reflectance transformation," (Eurographics symposium on rendering, Nicosia, Cyprus, 2006), pp. 26-28.

14. M. S. Drew, "Shape from color," Tech. Rep. CSS/LCCR TR 97-07 (1992).

15. M. L. Smith and L. N. Smith, "Dynamic photometric stereo - a new technique for moving surface analysis," in "Image Vision Computer," , vol. 23(9) (2005), vol. 23(9), pp. 841852.

16. M. S. Drew, "Photometric stereo without multiple images," Human Vision and Electronic Imaging 3016, 369-380 (1997).

17. G. Sharma, Digital color imaging handbook (CRC Press, 2003).

18. J. Lambert, Photometria (Augsburg, 1760).

19. B. Horn, "Height and gradient from shading," International Journal of computer vision 5, 37-75 (1990).

20. R. Klette and K. Schluns, "Height data from gradient fields," in "Machine Vision: Applications, Architectures and Systems Integration V," , vol. 2908 (SPIE, Boston, Massachusetts, USA, 1996), vol. 2908, pp. 204-215.

21. T. Wei and R. Klette, "Height from gradient using surface curvature and area constraints," in "Third Indian Conference on Computer Vision, Graphics and Image Processing (ICVGIP)," (Ahmedabad, India, 2002), pp. 204-210.

22. P. Kovesi, "Shapelets correlated with surface normals produce surfaces," in "IEEE International Conference on Computer Vision," , vol. 2 (IEEE, Beijing, China, 2005), vol. 2, pp. $994-1001$.

23. A. Woodward and P. Delmas, "Synthetic ground truth for comparison of gradient field integration methods for human faces," in "Image and Vision Computing New Zealand," (Dunedin, New Zealand, 2005).

24. A. Agrawal, R. Raskar, and R. Chellappa, "What is the range of surface reconstructions from a gradient field?" in "European Conference on Computer Vision (ECCV)," (2006), pp. $578-591$.

25. R. Frankot and R. Chellappa, "A method for enforcing integrability in shape from shading algorithms," IEEE Transactions on Pattern Analysis ans Machine Intelligence 10, 439-451 (1988).

26. K. Schlüns and R. Klette, Local and global integration of discrete vectors fields (Advances in Computer Vision, Wien, Austria, 1997), springer ed.

27. J. Y. Hardeberg, Acquisition and reproduction of color images: colorimetric and multispectral approaches (Universal Publishers/Dissertation.com, Parkland, Florida, 2001).

28. X. Huang, J. Brochard, D. Helbert, and M. Khoudeir, "Relief extraction of rough textured reflecting surface by image processing," in "IEEE/SPIE International Conference 
on Quality Control by Artificial Vision," (Le Creusot, France, 2007).

29. M. Oren and S. Nayar, "Generalization of the lambertian model and implications for machine vision," International Journal of Computer Vision 14, pp. 227-251 (1995).

30. D. Helbert, M. Khoudeir, and M.-T. Do, "Rough surfaces and relief extraction by generalized lambertian's photometric model," IEEE (IEEE International Conference on Signal Processing and Communication, Dubai, United Arab Emirates, 2007). 
Table 1. SNR error between the original surface and the recovered surface from three images or one color image.

\begin{tabular}{|c|c|c|c|c|}
\hline Surface & $5(\mathrm{a})$ & $\mathbf{5}(\mathrm{b})$ & $\mathbf{5}(\mathrm{c})$ & $\mathbf{5}(\mathrm{d})$ \\
\hline 3 images or 1 color image $(\mathrm{dB})$ & 36.109 & 51.982 & 61.112 & 46.045 \\
\hline
\end{tabular}

Table 2. Evolution of SNR between the reconstruction without noise and with noise as a function of the noise variance for surface 5 (a), in case of three gray level images or one color image.

\begin{tabular}{|c|c|c|c|}
\hline SNR of noise $(\mathrm{dB})$ & 15.513 & 9.374 & 8.105 \\
\hline 3 images $(\mathrm{dB})$ & 31.504 & 31.485 & 30.097 \\
\hline 1 color image $(\mathrm{dB})$ & 32.513 & 31.091 & 25.271 \\
\hline
\end{tabular}




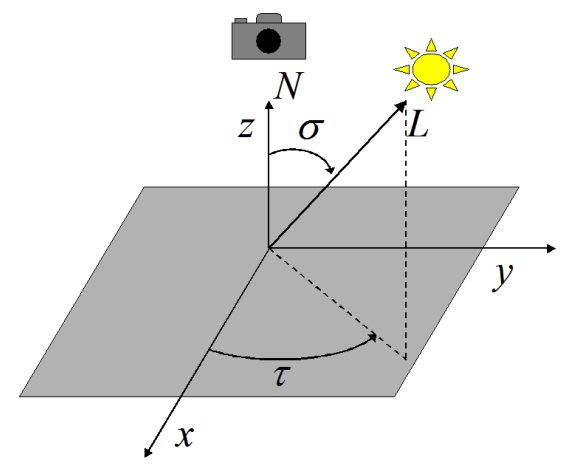

Fig. 1. Geometric definition of angles $\sigma$ and $\tau$.

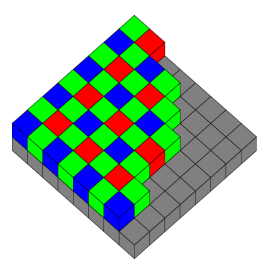

Fig. 2. (Color online) Example of a Bayer filter on a numerical camera. 


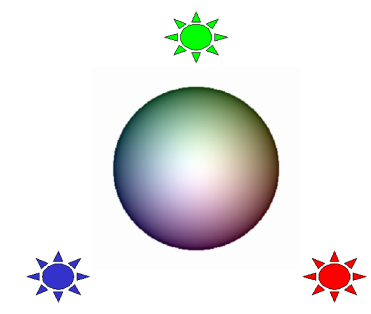

Fig. 3. (Color online) Example of a computer-created half-sphere lighted by three isolated colored sources of isolated light: a red one at $\tau_{1}=\frac{\pi}{3}$, a green one at $\tau_{2}=\pi$ and a blue one at $\tau_{3}=\frac{5 \pi}{3}$.

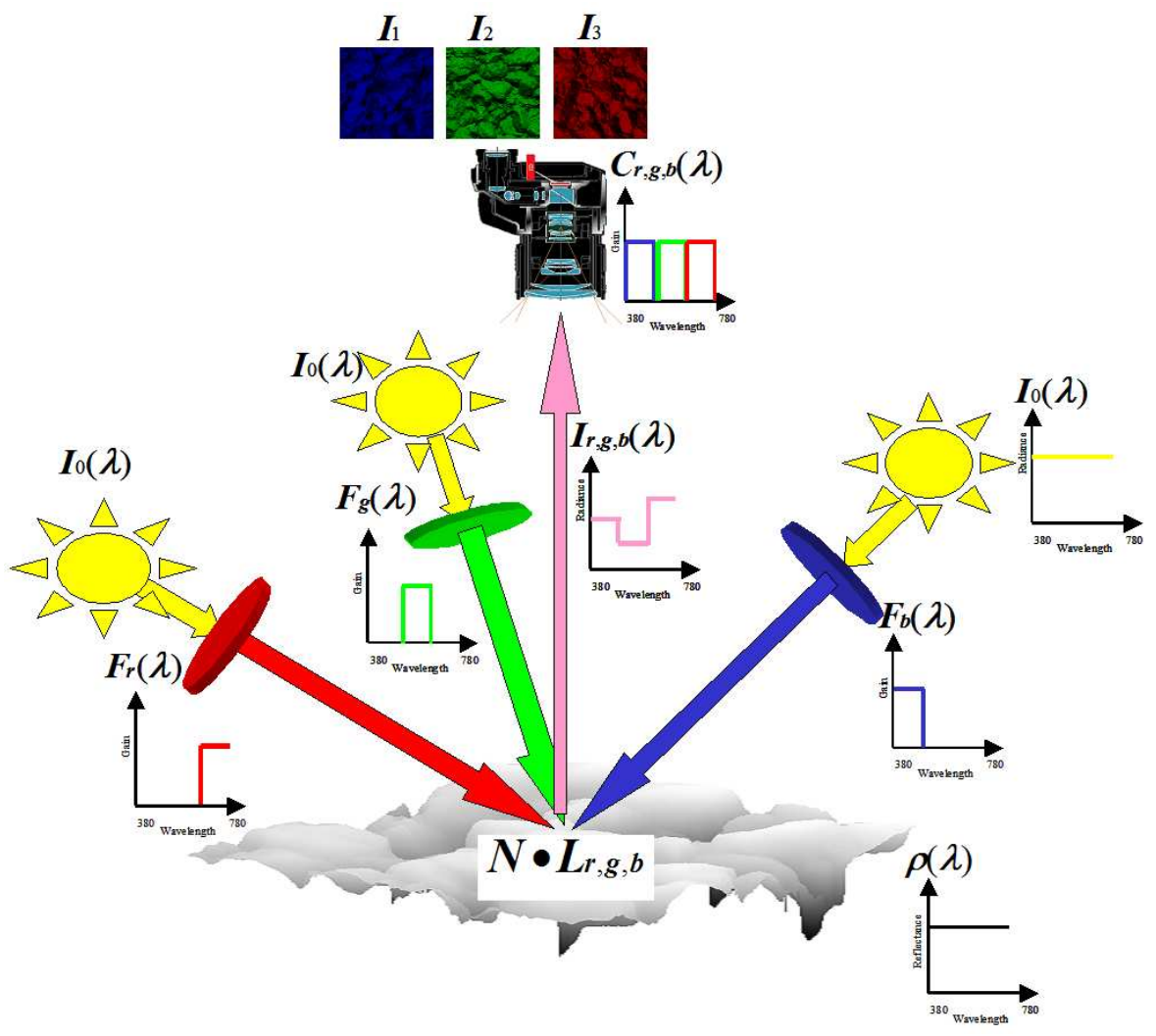

Fig. 4. (Color online) Color photometric stereo : three white lights with three different spectral filters illuminate the textured surface. For one facet, the reflected spectral radiance distribution depends on the normal direction. Then, the Bayer filter of the camera produces three isolated spectral images. Finally, the 3 -D surface can be recovered with these images and the photometric stereo theory. 


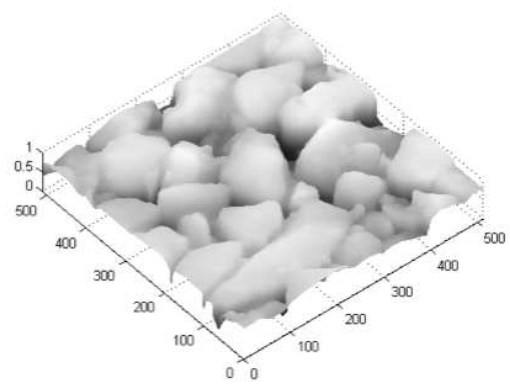

(a)

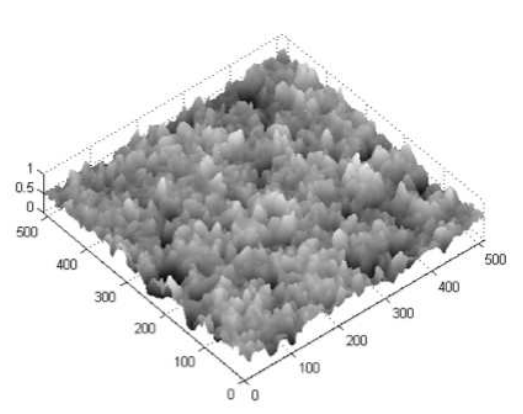

(c)

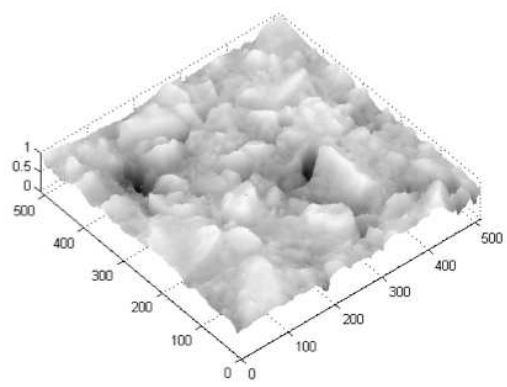

(b)

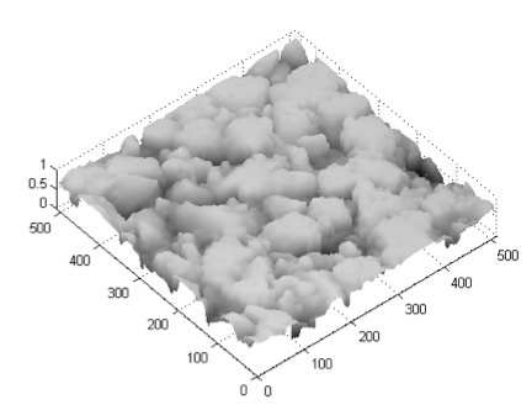

(d)

Fig. 5. 3-D surfaces acquired by a laser system.

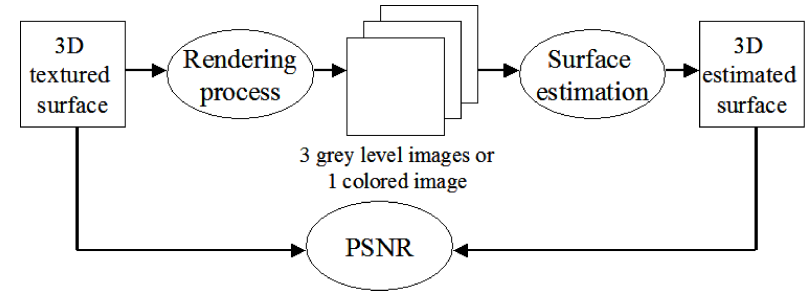

Fig. 6. Framework of the simulation. 

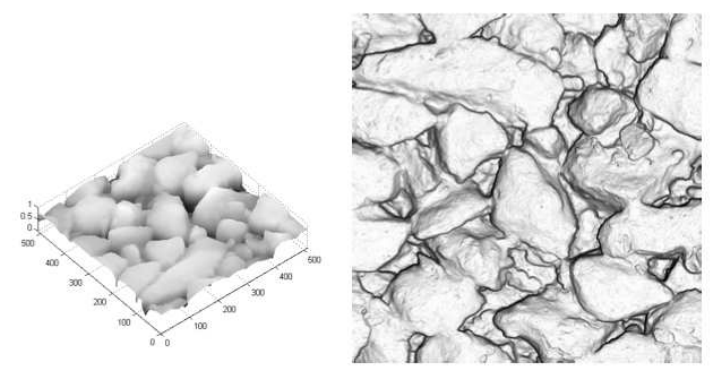

(a)

(b)

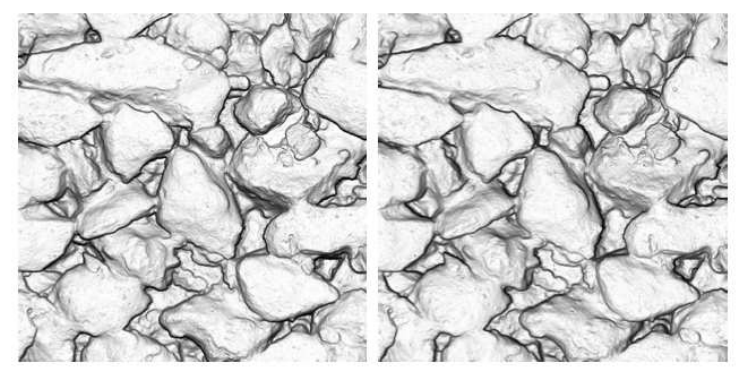

(c)

(d)

Fig. 7. Simulation of acquired images of a $3-\mathrm{D}$ textured surface illuminated according to Lambert's model. The zenith angle $\sigma$ is equal to $\frac{3 \pi}{70}$ (a: $3-\mathrm{D}$ representation, b: $\tau_{1}=\frac{\pi}{3}, \mathrm{c}: \tau_{2}=\pi$ and $\left.\mathrm{d}: \tau_{3}=\frac{5 \pi}{3}\right)$. 


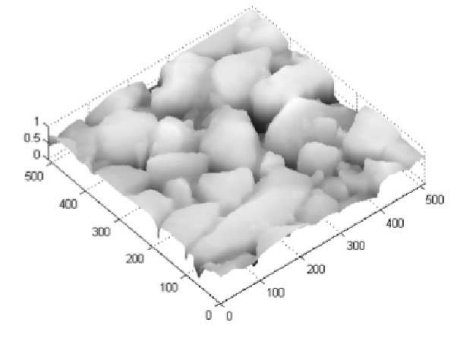

(a)

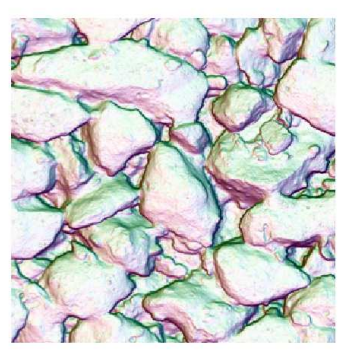

(b)

Fig. 8. (Color online) Simulation of acquired images of a 3-D textured surface illuminated by three colored lights with different azimuth angles (the blue light: $\tau_{b}=\frac{\pi}{3}$, the green light: $\tau_{g}=\pi$ and the red light: $\left.\tau_{r}=\frac{5 \pi}{3}\right)$ with Lambert's model. The zenith angle $\sigma$ is equal to $\frac{3 \pi}{70}$ (a: 3 -D representation and b: Colour image).

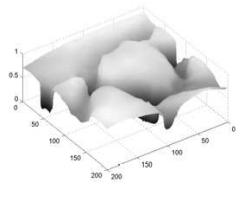

(a)

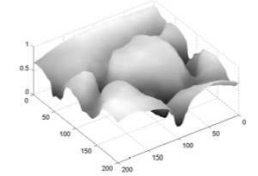

(b)

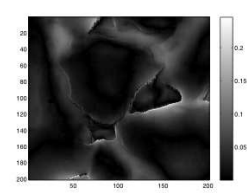

(c)

Fig. 9. Example of a part of original textured surface (figure 5) and the same part of the reconstructed surface (a: Original surface, b: Reconstructed surface and c: Error map). 


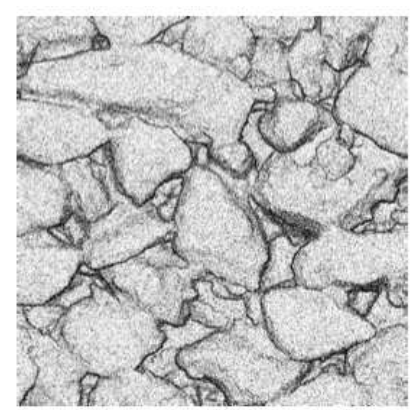

(a)

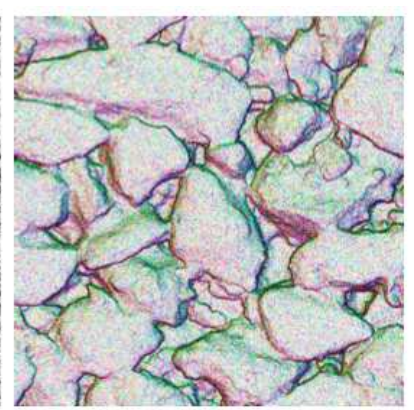

(b)

Fig. 10. (Color online) Illustration of noise addition for three gray level image acquisition (a) or one color image acquisition (b). In the case of three acquisitions of gray level images, only the azimuth angle $\tau=\pi$ is represented. The SNR is equal to 8.105 .

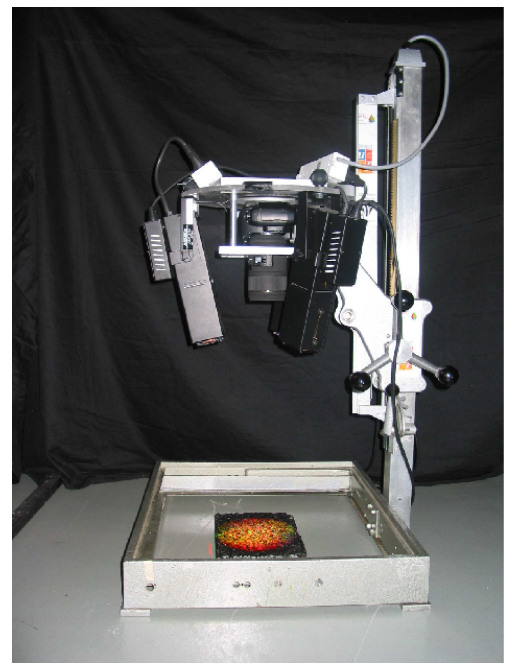

Fig. 11. (Color online) Acquisition system with the color lights. 


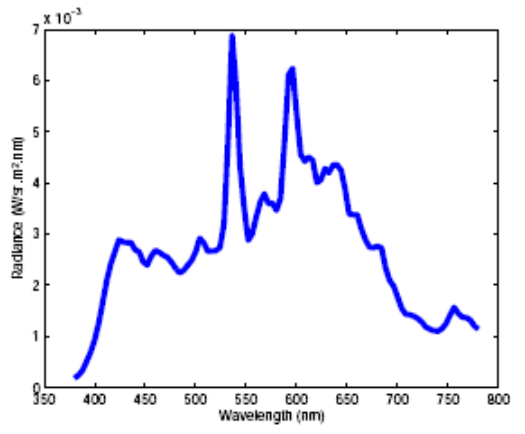

(a)

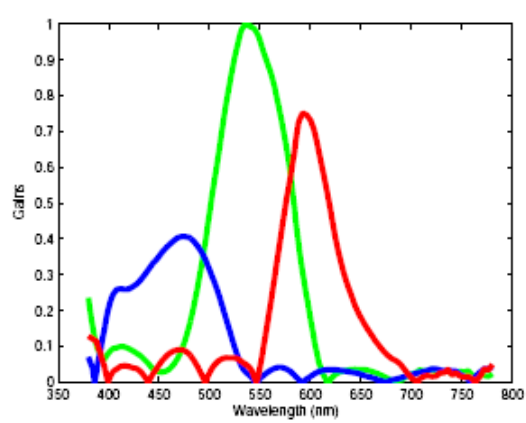

(c)

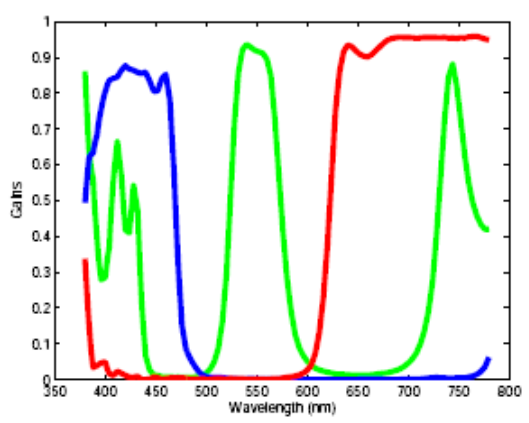

(b)

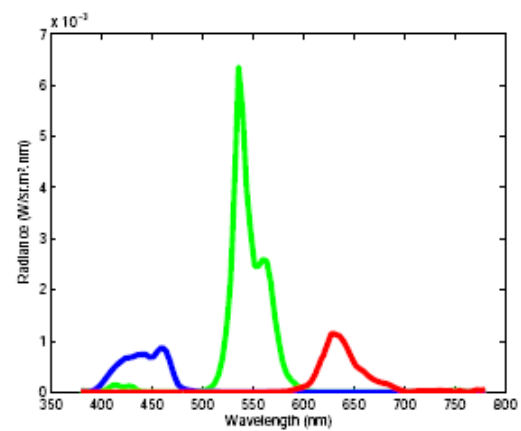

(d)

Fig. 12. (Color online) Spectral analysis of the 3-D reconstruction system (a: Gas-discharge lamp, b: Color filters, Bayer filter and d: Signal received).

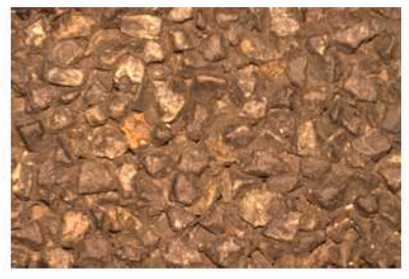

(a)

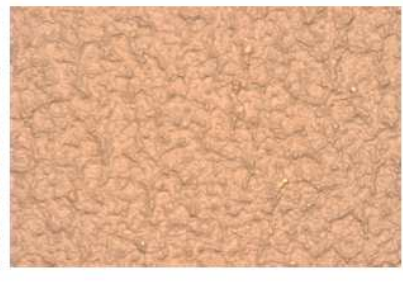

(b)

Fig. 13. Example of two real acquisition surfaces (a: road surface and b: stucco). 


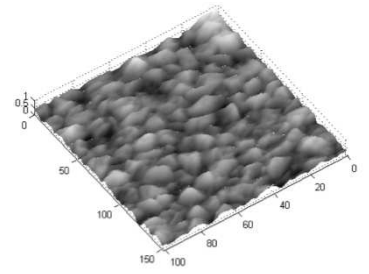

(a)

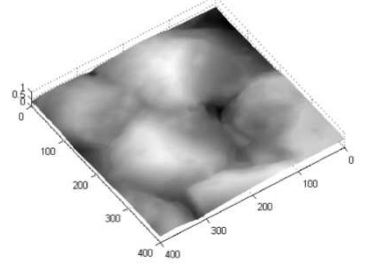

(c)

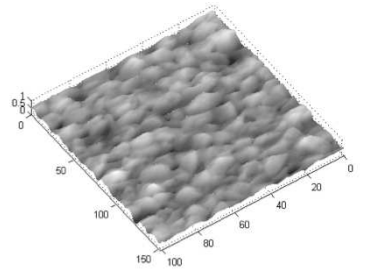

(b)

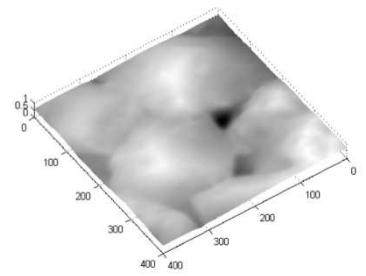

(d)

Fig. 14. Comparison between color photometric reconstruction and standard photometric reconstruction for the surface 13 (a: 3 images, b: Color image, c: Zoom for 3 images and d: Zoom for color image). 


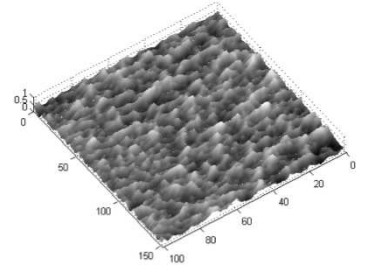

(a)

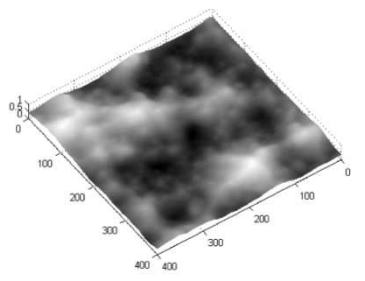

(c)

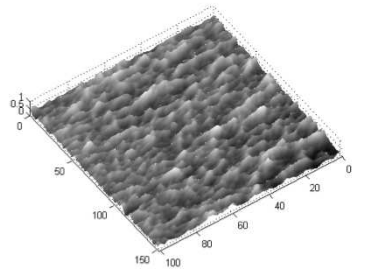

(b)

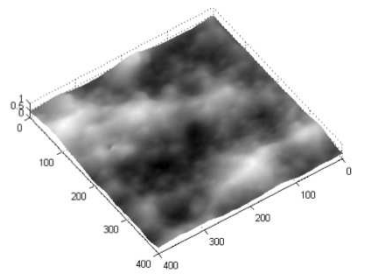

(d)

Fig. 15. Comparison between color photometric reconstruction and standard photometric reconstruction for the surface 13 (a: 3 images, b: Color image, c: Zoom for 3 images and d: Zoom for color image). 